\title{
Effect of ischemic compression performed by family caregivers on myofascial pain syndrome and the care burden of the families of patients: a multicenter open-label randomized comparative study
}

\author{
Hideaki Hasuo $^{1}$, Kotaro Hashimoto $^{2}$, Hanako Iwamoto ${ }^{3}$, Naoko Miwa $^{4}$, Toru Kawashima ${ }^{4}$, Akitoshi Oda ${ }^{5}$, \\ Go Sekimoto ${ }^{6}$ \\ ${ }^{1}$ Department of Psychosomatic Medicine, Kansai Medical University, Osaka, Japan; ${ }^{2}$ Department of Home Care, Fukushima Home Palliative Care \\ Clinic, Fukushima, Japan; ${ }^{3}$ Department of Home Care, Sakurashinmachi Urban Clinic, Tokyo, Japan; ${ }^{4}$ Department of Home Care, Komatsu Clinic, \\ Osaka, Japan; ${ }^{5}$ Department of Home Care, Home Clinic Kashiwa, Chiba, Japan; ${ }^{6}$ Department of Home Care, Sekimoto Clinic, Kobe, Japan \\ Contributions: (I) Conception and design: H Hasuo; (II) Administrative support: All authors; (III) Provision of study materials or patients: All authors; \\ (IV) Collection and assembly of data: H Hasuo, K Hashimoto, H Iwamoto, N Miwa, T Kawashima; (V) Data analysis and interpretation: H Hasuo; (VI) \\ Manuscript writing: All authors; (VII) Final approval of manuscript: All authors. \\ Correspondence to: Hideaki Hasuo, PhD. Department of Psychosomatic Medicine, Kansai Medical University, Shinmachi 2-3-1, Hirakata, Osaka 573- \\ 1090, Japan. Email: hasuohid@hirakata.kmu.ac.jp.
}

Background: Ischemic compression is a manual therapy technique for myofascial pain. This study aimed to verify the effect of ischemic compression performed by family caregivers on myofascial pain syndrome (MPS) in patients and on the family's care burden.

Methods: This multicenter, open-label, randomized, comparative study included patients with myofascial pain and their family caregivers who were randomized into the following groups: ischemia compression (performed by a family caregiver), sham ischemia compression, or untreated control. The effectiveness and safety of ischemic compression and the burden on family caregivers were evaluated. The primary endpoint was the rate of $50 \%$ or more improvement in the patient's mean numerical rating scale pain score in the previous 24 hours, 14 days after starting the intervention. The secondary endpoint was the rate of change in the family caregivers' reaction assessments.

Results: A total of 75 patients and caregivers (70 patients with cancer and family caregivers) who received home medical care were enrolled at three facilities. The study completion rate was $94.7 \%$, and there were no adverse events. The rate of $50 \%$ or more improvement in the numerical rating scale score was $64.0 \%$ in the ischemic compression group, $16.0 \%$ in the sham ischemic compression group, and $4.0 \%$ in the control group $(\mathrm{P}<0.001)$. Caregivers' self-esteem was significantly lower in the ischemic compression and sham ischemic compression groups than in the control group. However, there was no significant difference between the two groups $(\mathrm{P}=0.370)$.

Conclusions: Ischemic compression for myofascial pain in patients performed by family caregivers can increase the analgesic effect in patients and self-esteem in family caregivers.

Trial Registration: The University Hospital Medical Information Network Clinical Trials Registry (approval number: UMIN000036605).

Keywords: Ischemic compression; myofascial pain syndrome (MPS); family caregivers; analgesic effect; selfesteem

Submitted Aug 15, 2021. Accepted for publication Nov 12, 2021.

doi: $10.21037 /$ apm-21-2276

View this article at: https://dx.doi.org/10.21037/apm-21-2276

^ ORCID: 0000-0002-4689-1203. 


\section{Introduction}

Myofascial pain syndrome (MPS) is a functional disorder characterized by pain. The diagnostic criteria established by Rivers indicate that the myofascial trigger point (MTrP) needs to be palpated, and patient pain needs to be reproduced with palpation of the $M \operatorname{Tr} P$ (1). According to the US Centers for Disease Control and Prevention, three main conditions that cause daily life disability include heart disease, arthritis, and back pain (2). Of the patients with cancer, $11.9-45 \%$ complained of back pain and experienced MPS (3-5). To the best of our knowledge, there have been no reports on the prevalence of MPS in patients receiving home care. Continuous muscle contraction due to the same posture or postural restriction has been reported as a mechanism for MTrP formation (6). Patients receiving home care (especially patients with cancer) often have the same posture due to decreased activity, and the prevalence rate of MPS is expected to be high. In addition, the decreased activity is expected to be related to psychological stress (7). Psychological stress has been reported to be associated with low back pain in mice. One report found that more than half of patients with cancer have MPS with psychological stress (8).

No standard treatment has been established for patients with MPS (9). Clinically, trigger point injections of a local anesthetic into the MTrP, dry needling, acupuncture, and manual treatment are available treatment options (10-13). Ischemic compression is a manual therapy technique that inactivates MTrP by pressing it with a finger to make it ischemic. This ischemic compression has been reported to improve MPS pain and pressure pain thresholds within a short period (14-17). The less invasiveness related to the non-penetration and convenience has been identified as a feature of ischemic compression. Although it is common for family caregivers to perform ischemic compression in daily life, to our knowledge, no studies have suggested that ischemic compression performed by non-health workers is helpful.

Family caregivers experience great emotional distress due to the burden of caring. Decreased quality of life (QOL) and a high prevalence of psychiatric disorders among family caregivers have been reported (18). Family caregivers of patients with cancer feel guilty that they are not doing enough for their patients (19). The QOL of family caregivers during home palliative care is associated with self-esteem during care and affirmation of care $(20,21)$. One of the factors affecting the low QOL of family caregivers is the degree of patient distress (22). Thus, intervention by family caregivers to improve patient conditions can decrease the distress of caregivers (23).

We hypothesized that ischemic compression in areas involved in MPS by the family caregiver in patients receiving home care rapidly improves the pain and threshold for tenderness, thereby providing favorable effects on the self-esteem of the family caregiver. We present the following article in accordance with the CONSORT reporting checklist (available at https://apm.amegroups. com/article/view/10.21037/apm-21-2276/rc).

\section{Methods}

\section{Objective}

The objective of this study was to verify the effects of ischemic compression performed for MPS by family caregivers on (I) myofascial pain in patients and (II) on the care burden of the family.

\section{Study design}

We conducted a multicenter open-label randomized comparative study to assess the differences in patient interventions for MPS by family caregivers. The present study was conducted from 2019 to 2020 at three clinics in urban and rural areas of Japan. The study adhered to the principles of the Declaration of Helsinki (as revised in 2013). The study was approved by the Medical Ethics Committee of Kansai Medical University (reference number: 2019021) and informed consent was obtained from all patients. This study was registered with the University Hospital Medical Information Network Clinical Trials Registry (approval number: UMIN000036605) on May 11, 2019.

\section{Study participants}

Patients and their family caregivers who met the following eligibility criteria were included in the study: (I) received home medical care from the institutions that participated in this study; (II) met the Rivers' MPS diagnostic criteria (1); and (III) had MPS with a numerical rating scale (NRS) score of 3 or higher over the last 24 hours. MPS is diagnosed according to the following criteria: (I) a tender spot found on palpation with or without referral of pain; (II) recognition of pain symptoms by the patient on palpation of a tender spot; and at least three of the following: (III) muscle stiffness or 
spasm; (IV) limited range of motion of an associated joint; (V) pain that worsens with stress; and (VI) palpation of a taut band and/or nodule associated with a tender spot (1). The exclusion criteria included the following: (I) patients and family caregivers with mental disorders (e.g., mood disorder, cognitive impairment); (II) patients with rheumatoid pathologies (e.g., fibromyalgia, polymyalgia rheumatica); (III) patients and family caregivers younger than 20 years; and (IV) patients wanting to use superficial massage or new medication in the near future. Family caregivers were defined as the most direct caregivers in the family, such as relatives and spouses, and were limited to one person living together with the patient or visiting at least three times a week.

\section{Procedure}

Upon enrollment and after obtaining written informed consent, the study participants were randomly allocated to three groups using the permuted block method with a block size of 4 and a 1:1:1 allocation ratio for the three study groups: ischemic compression, sham compression, and control groups. Allocation and data management were performed by the Research Secretariat of Kansai Medical University, which was not involved in direct participant care. Each participant was automatically informed of their allocation after obtaining consent. Concurrently, clinicians responsible for the present study were automatically informed of the participant allocations. The allocation process was concealed by the clinicians involved in the statistical analysis. All participant data were anonymized.

In the ischemic compression group, a 30-s continuous compression with the thumb was performed three times by a family caregiver for the MTrP with a 30 -second interval between each repetition. The degree of continuous compression was defined as the maximum pressure at which the patient could tolerate the pressure pain, and the degree of compression increased when the pressure pain began to decrease. In the sham compression group, interventions that were similar to those of the ischemic compression group were performed, although the degree of continuous compression was the minimum pressure at which the patient felt pressure pain. In the control group, no specific therapy for $\mathrm{MTrP}$ was performed by family caregivers. In all groups, the patients were marked with ink at the location of the MTrPs by the clinicians. All groups were given a pamphlet with specific intervention details. The method of ischemic compression in this pamphlet was based on previous studies (14-17). For the ischemic compression and sham compression groups, direct instruction on the technique was given by the clinicians to family caregivers for approximately 5 minutes on day 0 (T0, the day before the start of the intervention). Patients were assessed by clinicians to ensure that they had been treated as indicated and properly on day 14 (T1, 14 days after the start of the intervention). During the intervention period, there was no use of superficial massage or new medication that could interfere with the response to the intervention in all groups. The study participants were not allowed to cross from one group to another until the end of the study. If a patient in the sham compression group or the control group desired ischemic compression, it was performed after the study period.

Ischemic and sham compressions for MPS at the two most painful areas were performed for patients by family caregivers three times a week for 2 weeks (six sessions in total). Among all groups, analgesic treatments for MPS were continued. Evaluations were performed at T0 and T1. The following information was obtained during the pre-observation period: patient demographic information, primary illness, Eastern Cooperative Oncology Group performance status (ECOG PS), number of MTrPs, duration of pain, analgesic drug use, personalized pain goal score, family caregiver demographic information, caregiver relationship with the patient, and duration of home medical care. The following information was obtained at T0 and T1: patient pain intensity score, pressure pain thresholds, adverse events, and family caregiver care burden score. The data were collected at the patients' homes by clinicians who were responsible for the present study.

In this study, ischemic compression was not performed within the last $24 \mathrm{~h}$ of the $\mathrm{T} 1$ evaluation.

\section{Measures}

\section{Pain intensity}

Patients evaluated their average pain intensity (PI) during the previous 24 hours using an 11-point NRS ranging from 0 (no pain) to 10 (worst possible pain) (24). Pain scores of 1-4 indicated mild PI, 5-6 indicated moderate PI, and 7-10 indicated severe PI (25). The reliability and validity of this scale were established (26). For multiple MPS areas with different NRS scores, the average NRS score was used. The best cutoff point for the NRS rate of change was reported to be $50 \%$ when determining the proportion of patients with clinically significant pain improvement (27). 


\section{Personalized pain goal}

One way to ensure that pain management goals are tailored to individual needs is to use a personalized pain goal (PPG), which relies on the patient criteria for meaningful pain relief. PPGs were assessed by asking patients the following question: "What is the maximum level of pain that would allow you to achieve comfort in physical, functional, and psychosocial domains?" Patients responded using 11-point NRS scores ranging from 0 (I feel comfortable and at ease at an NRS score of 0 points) to 10 (I feel comfortable even at an NRS score of 10 points). The median PPG score of patients with cancer was $3(28)$.

\section{Pressure pain thresholds}

A 10 -mm-diameter attachment to a digital ergometer (Digital force gages RZ series AIKOH, Osaka, Japan) was pressed against the skin on the patient's MTrP to measure the minimum pressure (pressure pain threshold; PPT) at the MTrP site at which the patient felt pain. PPT was measured twice, and the average score was used. The validity of measuring PPT at the MTrP site has been verified previously (29).

\section{Japanese version of the caregiver reaction assessment (CRA-J)}

The CRA-J is a multidimensional tool for assessing the care burden and a self-report questionnaire comprising 18 items scored with a rating scale of $1-5$. A high CRA-J score indicates a high burden of care. The CRA-J includes the following five domains: impact on schedule (five items), caregiver's self-esteem (five items), lack of family support (four items), impact on health (two items), and impact on finances (two items). The reliability and validity of the care burden for the families of home care patients have been verified, and it has a high internal consistency with a Cronbach's coefficient alpha of $0.73-0.89$ for each domain (30).

\section{Adverse events}

Adverse events were assessed using the Common Terminology Criteria for Adverse Events v.5.0 (Japan Clinical Oncology Group version) (31).

\section{Outcomes}

The primary endpoint was the rate of improvements of $50 \%$ or more in the mean NRS pain score of the patients within the last $24 \mathrm{~h} 14$ days after starting the intervention.
The secondary endpoints were the rate of change in the PPT for the patient's MTrP, the presence of adverse events, and the rate of change in the caregiver reaction assessment scores of family caregivers.

\section{Sample size calculation}

In a previous study (11), the average NRS change for cervicogenic headache originating from MTrP 2 weeks after the onset of treatment was $2.38 \pm 2.98$ [mean \pm standard deviation (SD)] for the ischemic compression group and $0.14 \pm 1.39$ for the control group. Based on this test, 60 participants ( $n=20$ in each group) were required when the significance level of the test was $5 \%$ on both sides, and the detection power was $90 \%$. Thus, 75 people were selected, taking into consideration a withdrawal rate of $20 \%$.

\section{Statistical analysis}

Data are reported as the mean and SD, median with interquartile range, or frequencies (\%) with the confidence interval (CI), as appropriate. We used the Kruskal-Wallis test and Pearson's chi-squared test for the following dependent variables: age, sex, ECOG PS, MPS site, number of MTrPs, duration of pain, PPT, NRS score (day 0), PPG score (day 0), analgesic drug use, and CRA-J score (day 0). The improvement of $50 \%$ or more in the NRS score before and after the intervention, which is the primary endpoint, was analyzed using Cochran's Q test. Changes in the NRS scores, PPT, and CRA-J scores were analyzed using the Wilcoxon signed-rank test. For comparisons of the groups, we used the time-course as the intra-participant factor and the group as the inter-participant factor for the Friedman test, and multiple comparisons were corrected using the Bonferroni method.

The main analysis was based on the intention-to-treat principle, and no study participants were excluded from the analysis. If participants withdrew from the study, the NRS scores, PPT, and CRA-J scores after withdrawal were replaced with scores just before withdrawal. The withdrawal cases included those for which the principal investigator decided that participation should be discontinued due to adverse events or progression of the underlying disease, the participant wished to discontinue participation, or a new analgesic was added during the study period.

Statistical significance was set at $\mathrm{P}<0.05$. Statistical analyses were conducted using SPSS version $25.0 \mathrm{~J}$ for 


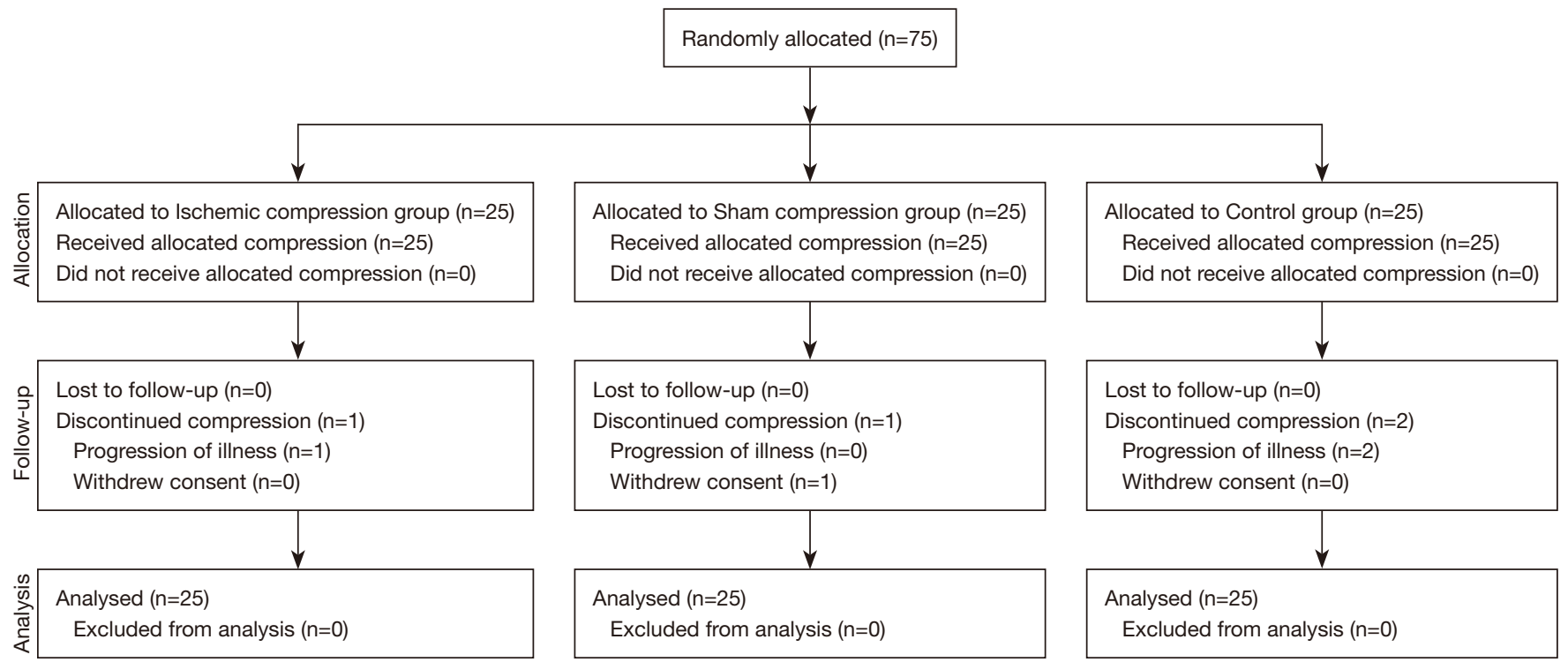

Figure 1 CONSORT diagram.

Macintosh (SPSS, Inc., IBM Corp., Chicago, IL, USA).

\section{Patient and public involvement}

Patients or the public were not involved in the design, conduct, reporting, or dissemination plans of our research.

\section{Results}

All 75 participants (70 were patients with cancer and family caregivers) were randomized, and 71 participants (94.7\%) completed the study (Figure 1). On T1, all patients who were followed up were confirmed by clinicians to have received appropriate interventions when indicated. Table 1 shows the demographic and clinical characteristics of the study participants. The demographic and clinical characteristics and measures for patients in the ischemic compression, sham compression, and control groups are presented in Table 2. No significant difference was noted in any measure between the groups. The MPS sites were found only in the upper and lower back, but not in other areas (Figure 2).

\section{Primary outcome analysis}

The rate of improvements of $50 \%$ or more in the NRS score for patients 14 days after the intervention was $64.0 \%$ (90\% CI: $47.9-80.1 \%$ ) for the ischemic compression group, $16.0 \%$ (90\% CI: 3.7-28.3) for the sham ischemic compression group, and $4.0 \%$ (90\% CI: $-2.5 \%$ to $10.5 \%$ ) for the control group $(\mathrm{P}<0.001$; Figure 3$)$. The changes in the mean NRS scores before and after the intervention for the three groups were compared, and the results are presented in Table 3. The ischemic compression group had significantly lower NRS scores at T1 than the sham compression and control groups $(\mathrm{P}=0.010$ and $\mathrm{P}<0.001$, respectively). There was no difference between the sham compression and control groups $(\mathrm{P}=0.407)$.

When limited to patients with cancer (70 patients), the rate of improvements of $50 \%$ or more in the NRS score for patients 14 days after the intervention was $60.9 \%$ (90\% CI: $43.8-78.0 \%)$ for the ischemic compression group, $8.7 \%$ (90\% CI: $-0.9 \%$ to $18.3 \%$ ) for the sham ischemic compression group, and $4.2 \%$ (90\% CI: $-2.6 \%$ to $11.0 \%$ ) for the control group $(\mathrm{P}<0.001)$.

\section{Secondary outcome analysis}

The mean pressure pain thresholds for the three groups before and after the intervention were compared, and the results are presented in Table 3. The ischemic compression group had a significantly higher pressure pain threshold at $\mathrm{T} 1$ than the sham compression and control groups $(\mathrm{P}<0.001$ for both). There was no difference between the sham compression and control groups $(\mathrm{P}=1.000)$. No adverse events were observed in any of the patients or family caregivers. 
Table 1 Demographic and clinical characteristics of study participants

\begin{tabular}{|c|c|}
\hline Characteristics & Values \\
\hline \multicolumn{2}{|l|}{ Patients } \\
\hline Age (years), mean (SD) & $75.3(12.1)$ \\
\hline \multicolumn{2}{|l|}{ Sex, n (\%) } \\
\hline Male & $41(54.7)$ \\
\hline Female & $34(45.3)$ \\
\hline Primary illness (cancer), n (\%) & $70(93.3)$ \\
\hline \multicolumn{2}{|l|}{ Primary cancer site, $\mathrm{n}$} \\
\hline Lung & 11 \\
\hline Gastrointestinal & 25 \\
\hline Liver, pancreas, biliary system & 13 \\
\hline Gynecological & 6 \\
\hline Urological & 6 \\
\hline Others & 9 \\
\hline Primary illness (non-cancer), n (\%) & $5(6.7)$ \\
\hline Old cerebral infarction & 2 \\
\hline Amyotrophic lateral sclerosis & 1 \\
\hline Chronic heart failure & 1 \\
\hline Liver cirrhosis & 1 \\
\hline \multicolumn{2}{|l|}{ ECOG PS, n (\%) } \\
\hline $0-2$ & $12(16.0)$ \\
\hline $3-4$ & $63(84.0)$ \\
\hline \multicolumn{2}{|l|}{ Family caregivers } \\
\hline Age (years), mean (SD) & $62.4(14.0)$ \\
\hline \multicolumn{2}{|l|}{ Sex, n (\%) } \\
\hline Male & $20(26.7)$ \\
\hline Female & $55(73.3)$ \\
\hline \multicolumn{2}{|l|}{ Relationship with the patients, $\mathrm{n}(\%)$} \\
\hline Wife & $29(38.7)$ \\
\hline Husband & $11(14.7)$ \\
\hline Daughter & $19(25.3)$ \\
\hline Son & $6(8.0)$ \\
\hline Mother & $2(2.7)$ \\
\hline Others & $8(10.7)$ \\
\hline
\end{tabular}

Table 1 (continued)
Table 1 (continued)

\begin{tabular}{lc}
\hline Characteristics & Values \\
\hline Duration of home medical care (months), mean (SD) & $3.7(9.3)$ \\
CRA-J score, mean (SD) & \\
Impact on schedule & $16.9(4.9)$ \\
Caregiver's self-esteem & $16.2(3.8)$ \\
Lack of family support & $9.0(4.6)$ \\
Impact on health & $6.7(3.1)$ \\
Impact on finances & $6.3(3.2)$ \\
\hline
\end{tabular}

SD, standard deviation; ECOG PS, Eastern Cooperative Oncology Group performance status; CRA-J, Japanese version of the Caregiver Reaction Assessment.

The mean CRA-J scores before and after the intervention were compared among the three groups, and the results are presented in Table 4. The ischemic compression and sham compression groups showed significantly lower caregiver self-esteem scores (higher caregiver self-esteem) at $\mathrm{T} 1$ than the control group $(\mathrm{P}<0.001$ and $\mathrm{P}=0.010)$, but there was no significant difference between the ischemic compression and sham compression groups $(\mathrm{P}=0.370)$. When limited to patients with cancer (70 family caregivers), the ischemic compression and sham compression groups showed significantly lower caregiver self-esteem scores (higher caregiver self-esteem) at $\mathrm{T} 1$ than the control group $(\mathrm{P}<0.001$ and $\mathrm{P}=0.005$ ), but no significant difference was noted between the ischemic compression and sham compression groups $(\mathrm{P}=0.349)$.

\section{Discussion}

To the best of our knowledge, this study is the first to evaluate the analgesic effect of ischemic compression by family caregivers of patients with myofascial pain and its effect on the care burden of the family.

The results of this study provide two important perspectives. First, ischemic compression for MPS by family caregivers was found to be useful for intractable pain associated with MPS. The reported pain severity was moderate among patients (median NRS score, 6.0) (25), and PPG was not achieved. The average pain duration of MPS was 6 months or more, which suggests chronic pain (32). Patients receiving home care, especially patients with cancer, 
Table 2 Comparison of the demographic characteristics, clinical characteristics, and measures between the ischemic compression, sham compression, and control groups

\begin{tabular}{|c|c|c|c|c|}
\hline Characteristics & $\begin{array}{l}\text { Ischemic compression group } \\
\qquad(\mathrm{n}=25)\end{array}$ & $\begin{array}{l}\text { Sham compression group } \\
\qquad(\mathrm{n}=25)\end{array}$ & $\begin{array}{l}\text { Control group } \\
\qquad(n=25)\end{array}$ & $P$ value \\
\hline Age (years), mean (SD) & $78.6(8.9)$ & $74.4(13.9)$ & $72.8(12.6)$ & 0.217 \\
\hline ECOG PS, n (\%) & & & & 0.226 \\
\hline Site of MPS, n (\%) & & & & 0.533 \\
\hline Upper back & $9(36.0)$ & $12(48.0)$ & $11(44.0)$ & \\
\hline Lower back & $16(64.0)$ & $13(52.0)$ & $14(56.0)$ & \\
\hline NRS score (day 0), median [IQR] & $7[5-7.5]$ & $7[5-8.5]$ & $5[5-8]$ & 0.400 \\
\hline PPG score, median [IQR] & $3[2-4]$ & 3 [2-3] & $2[2-3]$ & 0.373 \\
\hline \multicolumn{5}{|l|}{ Analgesic drug use, n (\%) } \\
\hline None & $8(32.0)$ & $12(48.0)$ & $7(28.0)$ & 0.305 \\
\hline Use & $17(68.0)$ & $13(52.0)$ & $18(72.0)$ & \\
\hline Opioid & $12(48.0)$ & $8(32.0)$ & $10(40.0)$ & 0.524 \\
\hline \multicolumn{5}{|l|}{ Family caregivers } \\
\hline Lack of family support & $8.4(3.3)$ & $9.8(5.7)$ & $8.8(4.5)$ & 0.544 \\
\hline Impact on health & $7.2(2.0)$ & $6.9(4.4)$ & $6.2(2.4)$ & 0.531 \\
\hline Impact on finances & $5.3(2.7)$ & $6.3(2.2)$ & $6.3(3.2)$ & 0.094 \\
\hline
\end{tabular}

SD, standard deviation; ECOG PS, Eastern Cooperative Oncology Group performance status; MPS, myofascial pain syndrome; PPT, pressure pain thresholds; PPG, personalized pain goal; NRS, numerical rating scale; IQR, interquartile range; CRA-J, Japanese version of the Caregiver Reaction Assessment.

have less time to live or less physical strength, and it is remarkable that the moderate and chronic pain improved significantly during the 2 weeks of intervention. A systematic review of ischemic compression by clinicians for shoulder pain showed immediate and short-term positive effects (33). In addition, ischemic compression was not performed within the last 24 hours before the T1 evaluation in this study, which suggests that this significant improvement was not a transient effect that occurred only during the ischemic compression, but there was a continuous effect. During the T1 evaluation, the PPT at the MTrP site was significantly higher for the ischemic compression group. This result suggests that ischemic compression may have an analgesic effect by inactivating MTrP. The mechanism of PPT-related 

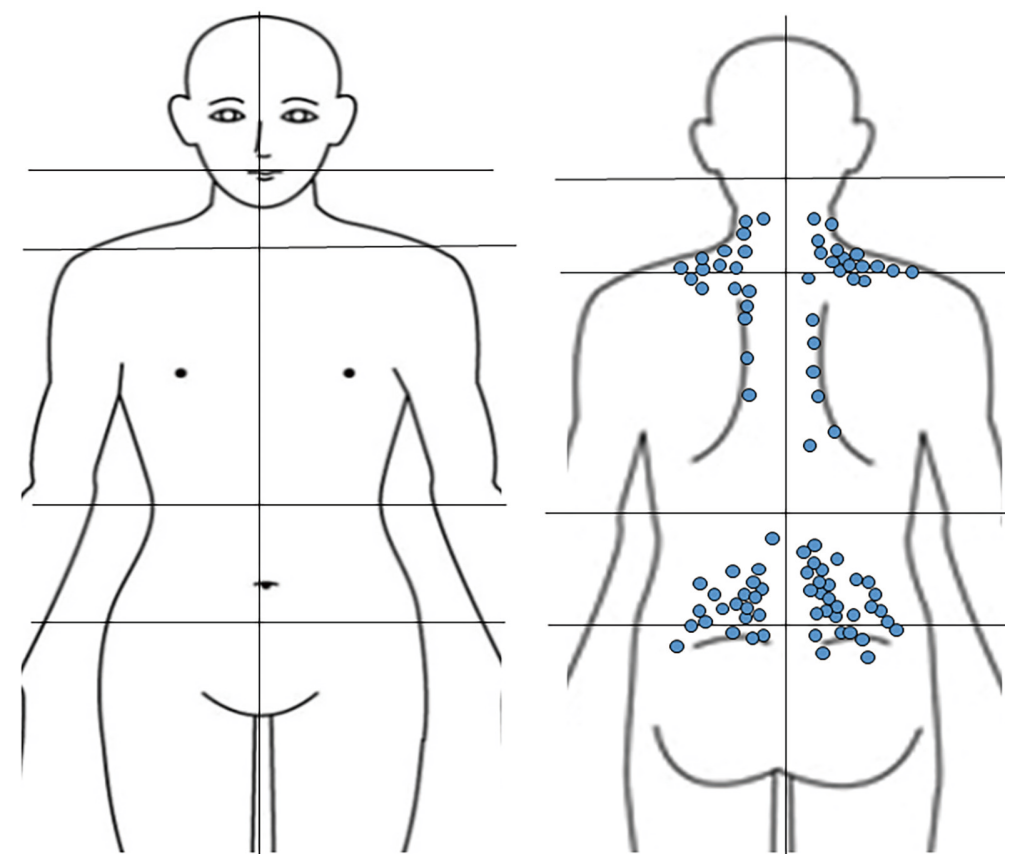

Figure 2 The MPS site (dots). MPS, myofascial pain syndrome.

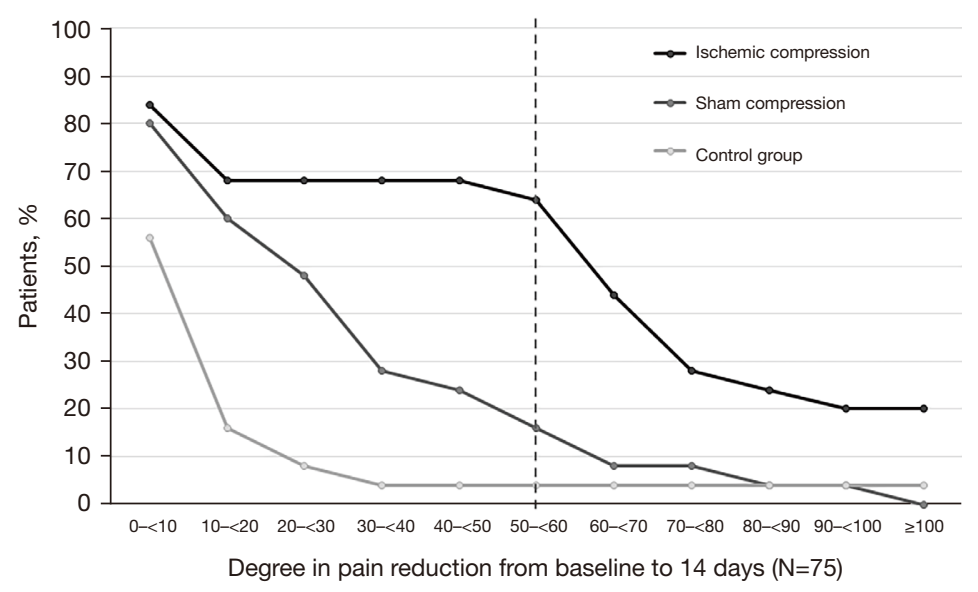

Figure 3 The percentage of patients achieving various levels of pain reduction from baseline to 14 days.

Table 3 Change in the numerical rating scale scores and the pressure pain threshold before and after intervention between the ischemic compression, sham compression, and control groups

\begin{tabular}{|c|c|c|c|c|c|c|c|c|c|c|c|}
\hline \multirow{2}{*}{ Characteristics } & \multicolumn{3}{|c|}{ Ischemic compression group (a) } & \multicolumn{3}{|c|}{ Sham compression group (b) } & \multicolumn{3}{|c|}{ Control group (c) } & \multirow{2}{*}{ - $P$ value } & \multirow{2}{*}{$\begin{array}{c}\text { Multiple } \\
\text { comparison }\end{array}$} \\
\hline & T0 & $\mathrm{T} 1$ & $P$ value & TO & $\mathrm{T} 1$ & $P$ value & TO & $\mathrm{T} 1$ & $P$ value & & \\
\hline NRS score, mean (SD) & $6.2(1.9)$ & $3.5(2.7)$ & $<0.001$ & $6.8(2.0)$ & $5.4(2.3)$ & 0.015 & $6.1(2.1)$ & $6.4(2.5)$ & 0.574 & 0.005 & $\begin{array}{l}a>b^{\star} \\
a>c^{\star \star \star}\end{array}$ \\
\hline PPT (kPa), mean (SD) & $23.9(9.1)$ & $35.8(13.5)$ & $<0.001$ & $24.4(6.9)$ & $23.5(6.2)$ & 0.166 & $25.7(6.7)$ & $24.3(8.4)$ & 0.164 & $<0.001$ & $\begin{array}{l}a>b^{\star \star \star} \\
a>c^{\star \star \star}\end{array}$ \\
\hline
\end{tabular}

${ }^{*}, \mathrm{P}<0.05 ;{ }^{* \star \star}, \mathrm{P}<0.001$. NRS, numerical rating scale; PPT, pressure pain threshold; SD, standard deviation. 
Table 4 The Japanese version of the Caregiver Reaction Assessment scores before and after the intervention between the ischemic compression, sham compression, and control groups

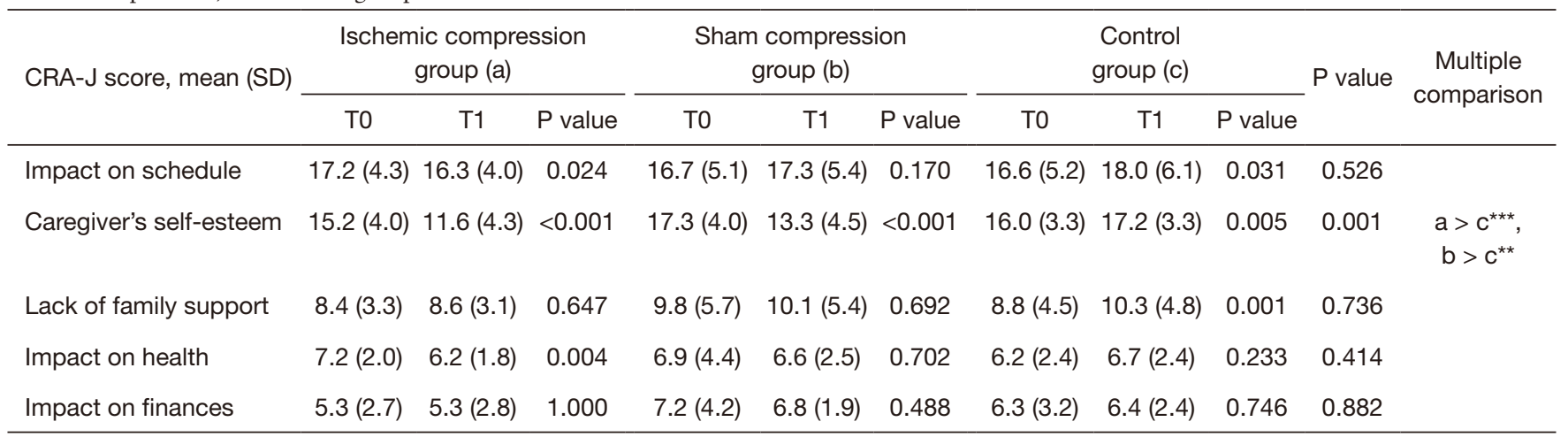

${ }^{* *}, \mathrm{P}<0.05 ;{ }^{* * *}, \mathrm{P}<0.001$. CRA-J, Japanese version of the Caregiver Reaction Assessment; SD, standard deviation.

inactivation is unknown, but it may reduce the nociception generated by MTrP.

This study cannot be compared with others because there are no other reports on ischemic compression performed by family caregivers. Furthermore, our search revealed no reports of ischemic compression for MPS performed by medical staff for home care or patients with cancer. There was a report of four sessions of ischemic compression performed by medical staff within 2 weeks for a cervicogenic headache originating from MPS with an intergroup change score of 2.55 (95\% CI: 0.80-4.30) (14), which is similar to the findings of this study. In this study, the rate of improvements of $50 \%$ or more improvement in the average NRS pain score in the patients during the previous 24 hours and 14 days after the onset of the intervention was very high (64.0\%). An improvement of $33 \%$ or more in the NRS pain score a day after the local anesthetic trigger point injection in patients with cancer was reported to be $59 \%$, and the rate of improvement in the NRS pain score of $50 \%$ or more after 1 week was $48.8 \%(4,8)$.

Second, the self-esteem of caregivers, which is a domain of the CRA-J for family caregivers, showed that the burdens for the ischemic compression group and the sham ischemic compression group were significantly reduced compared with that for the control group, although there was no significant difference between the two groups. The selfesteem of caregivers has been reported to play a role in increasing satisfaction in the relationship with end-of-life patients (21), while care stress shows a negative correlation with the self-esteem of caregivers (34). In this context, it is significant that the intervention by the family caregiver to improve the patient's symptoms increased their self- esteem about caring for the family member. Family caregiver interventions to improve the patient's condition have been reported to reduce family distress (23), while the self-esteem of the caregiver did not show a significant difference between the ischemic compression and sham compression groups, in which the patient's analgesia was and was not observed, respectively. This result suggests that increased caregiver self-esteem may be poorly correlated with the symptom relief of the patient, which results in improvements of the patient's condition. One study reported that the holding of the hands of patients with cancer by family caregivers did not significantly increase the patient's autonomic functioning, but significantly increased the family caregiver's autonomic functioning, resulting in family self-care (35).

Despite concerns about the physical burden on family caregivers due to ischemic compression, there was no negative effect on their health, which is one of the domains of the CRA-J. The patients in this study were older, and most patients had advanced cancer with a decreased ECOG PS. Therefore, it was expected that the care burden of family caregivers would be high, although the degree of the care burden remains unknown because the CRA-J has no cutoff value. Care for patients with chronic illnesses is associated with a high care burden and chronic stress for caregivers (36). In contrast, the physical condition of family caregivers of patients with cancer was reported to be more associated with their subjective care burden than with the severity of their condition (37). The protocol for ischemic compression by family caregivers remains undetermined; however, three sessions per week are considered appropriate because of the usefulness of this study for patients and 
the low burden on family caregivers. For the duration of ischemic compression, it has been reported that there is no difference in the effect between 30 and $60 \mathrm{~s}$ (15).

The limitations of our study are as follows: (I) the procedure was not consistent because the interventions were administered by family caregivers and non-supervised by clinicians; (II) the results cannot be generalized because homecare patients are mostly patients with cancer (however, the results of the main outcome were similar even when the study was limited to patients with cancer and their family caregivers); (III) there was selection bias due to the eligibility assessment, which was based on non-continuous registration; (IV) it was not possible to evaluate changes in analgesia over time through the six sessions of the ischemic compression because there were only two evaluation points; (V) it was not possible to compare the effects on different sites of MPS due to the small number of cases in this study; and (VI) there is no previous study on the interventions by family caregivers to improve the patient conditions for their self-esteem, and we were unable to compare the results of our study with others.

\section{Conclusions}

Ischemic compression for myofascial pain performed by family caregivers can increase the analgesia for patients and the self-esteem of family caregivers.

\section{Acknowledgments}

The authors thank the Research Secretariat of the Kansai Medical University Hospital. We also thank Jodi Smith, PhD ELS, from Edanz Group (https://en-author-services. edanz.com/ac) for editing the draft of this manuscript.

Funding: This work was supported by the Sasakawa Memorial Health Foundation.

\section{Footnote}

Reporting Checklist: The authors have completed the CONSORT reporting checklist. Available at https://apm. amegroups.com/article/view/10.21037/apm-21-2276/rc

Data Sharing Statement: Available at https://apm.amegroups. com/article/view/10.21037/apm-21-2276/dss

Peer Review File: Available at https://apm.amegroups.com/ article/view/10.21037/apm-21-2276/prf
Conflicts of Interest: All authors have completed the ICMJE uniform disclosure form (available at https://apm. amegroups.com/article/view/10.21037/apm-21-2276/coif) and report that this work was supported by the Sasakawa Memorial Health Foundation. The authors have no other conflicts of interest to declare.

Etbical Statement: The authors are accountable for all aspects of the work in ensuring that questions related to the accuracy or integrity of any part of the work are appropriately investigated and resolved. The study adhered to the principles of the Declaration of Helsinki (as revised in 2013). The study was approved by the Medical Ethics Committee of Kansai Medical University (reference number: 2019021) and informed consent was obtained from all patients.

Open Access Statement: This is an Open Access article distributed in accordance with the Creative Commons Attribution-NonCommercial-NoDerivs 4.0 International License (CC BY-NC-ND 4.0), which permits the noncommercial replication and distribution of the article with the strict proviso that no changes or edits are made and the original work is properly cited (including links to both the formal publication through the relevant DOI and the license). See: https://creativecommons.org/licenses/by-nc-nd/4.0/.

\section{References}

1. Rivers WE, Garrigues D, Graciosa J, et al. Signs and Symptoms of Myofascial Pain: An International Survey of Pain Management Providers and Proposed Preliminary Set of Diagnostic Criteria. Pain Med 2015;16:1794-805.

2. Center for Disease Control and Prevention (CDC). Disability and Health. Atlanta, GA: CDC, 2011. Available online: https://www.cdc.gov/ncbddd/disabilityandhealth/ healthyliving.html. Accessed July 1, 2021.

3. Thottungal A, Kumar P, Bhaskar A. Interventions for myofascial pain syndrome in cancer pain: recent advances: why, when, where and how. Curr Opin Support Palliat Care 2019;13:262-9.

4. Hasuo H, Kanbara K, Abe T, et al. Factors Associated with the Efficacy of Trigger Point Injection in Advanced Cancer Patients. J Palliat Med 2017;20:1085-90.

5. Ishiki H, Kinkawa J, Watanabe A, et al. Prevalence of myofascial pain syndrome in patients with incurable cancer. J Bodyw Mov Ther 2018;22:328-32.

6. Saxena A, Chansoria M, Tomar G, et al. Myofascial pain 
syndrome: an overview. J Pain Palliat Care Pharmacother 2015;29:16-21.

7. La Porta C, Tappe-Theodor A. Differential impact of psychological and psychophysical stress on low back pain in mice. Pain 2020;161:1442-58.

8. Hasuo H, Ishiki H, Matsuoka H, et al. Clinical Characteristics of Myofascial Pain Syndrome with Psychological Stress in Patients with Cancer. J Palliat Med 2021;24:697-704.

9. Fleckenstein J, Zaps D, Rüger LJ, et al. Discrepancy between prevalence and perceived effectiveness of treatment methods in myofascial pain syndrome: results of a cross-sectional, nationwide survey. BMC Musculoskelet Disord 2010;11:32.

10. Scott NA, Guo B, Barton PM, et al. Trigger point injections for chronic non-malignant musculoskeletal pain: a systematic review. Pain Med 2009;10:54-69.

11. Liu L, Huang QM, Liu QG, et al. Evidence for Dry Needling in the Management of Myofascial Trigger Points Associated With Low Back Pain: A Systematic Review and Meta-Analysis. Arch Phys Med Rehabil 2018;99:144-152.e2.

12. Li X, Wang R, Xing X, et al. Acupuncture for Myofascial Pain Syndrome: A Network Meta-Analysis of 33 Randomized Controlled Trials. Pain Physician 2017;20:E883-902.

13. Falsiroli Maistrello L, Geri T, Gianola S, et al. Effectiveness of Trigger Point Manual Treatment on the Frequency, Intensity, and Duration of Attacks in Primary Headaches: A Systematic Review and Meta-Analysis of Randomized Controlled Trials. Front Neurol 2018;9:254.

14. Jafari M, Bahrpeyma F, Togha M. Effect of ischemic compression for cervicogenic headache and elastic behavior of active trigger point in the sternocleidomastoid muscle using ultrasound imaging. J Bodyw Mov Ther 2017;21:933-9.

15. Kim SA, Oh KY, Choi WH, et al. Ischemic compression after trigger point injection affect the treatment of myofascial trigger points. Ann Rehabil Med 2013;37:541-6.

16. Behrangrad S, Kamali F. Comparison of ischemic compression and lumbopelvic manipulation as trigger point therapy for patellofemoral pain syndrome in young adults: A double-blind randomized clinical trial. J Bodyw Mov Ther 2017;21:554-64.

17. Rangon FB, Koga Ferreira VT, Rezende MS, et al. Ischemic compression and kinesiotherapy on chronic myofascial pain in breast cancer survivors. J Bodyw Mov Ther 2018;22:69-75.
18. Cameron JI, Franche RL, Cheung AM, et al. Lifestyle interference and emotional distress in family caregivers of advanced cancer patients. Cancer 2002;94:521-7.

19. Hileman JW, Lackey NR, Hassanein RS. Identifying the needs of home caregivers of patients with cancer. Oncol Nurs Forum 1992;19:771-7.

20. Ito E, Tadaka E. Quality of life among the family caregivers of patients with terminal cancer at home in Japan. Jpn J Nurs Sci 2017;14:341-52.

21. Mroz EL, Poulin MJ, Grant PC, et al. Caregiver SelfEsteem as a Predictor of Patient Relationship Satisfaction: A Longitudinal Study. J Palliat Med 2018;21:376-9.

22. Kim Y, Given BA. Quality of life of family caregivers of cancer survivors: across the trajectory of the illness. Cancer 2008;112:2556-68.

23. Spillers RL, Wellisch DK, Kim Y, et al. Family caregivers and guilt in the context of cancer care. Psychosomatics 2008;49:511-9.

24. Caraceni A, Cherny N, Fainsinger R, et al. Pain measurement tools and methods in clinical research in palliative care: recommendations of an Expert Working Group of the European Association of Palliative Care. J Pain Symptom Manage 2002;23:239-55.

25. Serlin RC, Mendoza TR, Nakamura Y, et al. When is cancer pain mild, moderate or severe? Grading pain severity by its interference with function. Pain 1995;61:277-84.

26. Hawker GA, Mian S, Kendzerska T, et al. Measures of adult pain: Visual Analog Scale for Pain (VAS Pain), Numeric Rating Scale for Pain (NRS Pain), McGill Pain Questionnaire (MPQ), Short-Form McGill Pain Questionnaire (SF-MPQ), Chronic Pain Grade Scale (CPGS), Short Form-36 Bodily Pain Scale (SF-36 BPS), and Measure of Intermittent and Constant Osteoarthritis Pain (ICOAP). Arthritis Care Res (Hoboken) 2011;63 Suppl 11:S240-52.

27. Smith SM, Dworkin RH, Turk DC, et al. Interpretation of chronic pain clinical trial outcomes: IMMPACT recommended considerations. Pain 2020;161:2446-61.

28. Dalal S, Hui D, Nguyen L, et al. Achievement of personalized pain goal in cancer patients referred to a supportive care clinic at a comprehensive cancer center. Cancer 2012;118:3869-77.

29. Reeves JL, Jaeger B, Graff-Radford SB. Reliability of the pressure algometer as a measure of myofascial trigger point sensitivity. Pain 1986;24:313-21.

30. Misawa T, Miyashita M, Kawa M, et al. Validity and reliability of the Japanese version of the Caregiver Reaction 
Assessment Scale (CRA-J) for community-dwelling cancer patients. Am J Hosp Palliat Care 2009;26:334-40.

31. Japan Clinical Oncology Group, Common terminology criteria for adverse events version 5.0 (CTCAE v5.0JCOG). Available online: http://www.jcog.jp/doctor/tool/ CTCAEv5J_20180730_v21_0.pdf. Accessed May 2, 2019.

32. Dworkin RH, Turk DC, Peirce-Sandner S, et al. Research design considerations for confirmatory chronic pain clinical trials: IMMPACT recommendations. Pain 2010;149:177-93.

33. da Silva AC, De Noronha M, Liberatori-Junior RM, et al. The Effectiveness of Ischemic Compression Technique on Pain and Function in Individuals With Shoulder Pain: A Systematic Review. J Manipulative Physiol Ther 2020;43:234-46.

Cite this article as: Hasuo $\mathrm{H}$, Hashimoto $\mathrm{K}$, Iwamoto $\mathrm{H}$, Miwa N, Kawashima T, Oda A, Sekimoto G. Effect of ischemic compression performed by family caregivers on myofascial pain syndrome and the care burden of the families of patients: a multicenter open-label randomized comparative study. Ann Palliat Med 2022;11(4):1179-1190. doi: 10.21037/apm-21-2276
34. Kim D. Relationships between Caregiving Stress, Depression, and Self-Esteem in Family Caregivers of Adults with a Disability. Occup Ther Int 2017;2017:1686143.

35. Sakuma H, Hasuo H, Fukunaga M. Effect of handholding on heart rate variability in both patients with cancer and their family caregivers: a randomized crossover study. Biopsychosoc Med 2021;15:14.

36. Vitaliano PP, Zhang J, Scanlan JM. Is caregiving hazardous to one's physical health? A meta-analysis. Psychol Bull 2003;129:946-72.

37. Kim Y, Carver CS, Shaffer KM, et al. Cancer caregiving predicts physical impairments: roles of earlier caregiving stress and being a spousal caregiver. Cancer 2015;121:302-10. 